\title{
A NOTE ON CLOSED MAPS AND METRIZABILITY
}

\section{M. HYMAN}

1. Introduction. Investigating conditions under which quotients of metric spaces are metrizable, Stone [3] and Morita and Hanai [2] independently obtained the following result.

Theorem A. If a topological space $Y$ is the closed, continuous image of a metric space, and if $Y$ satisfies the first axiom of countability, then $Y$ is metrizable.

The hypothesis of first-countability cannot be dropped. For example, if $Y$ is the quotient space obtained from the real line $R$ by identifying the integers to a point, then the natural projection

$$
\pi: R \rightarrow Y
$$

is closed and continuous, but $Y$ is not metrizable. Notice that, although $\pi$ is closed, the product map

$$
\pi \times \pi: R \times R \rightarrow Y \times Y
$$

is not closed. (For example, the set $\{(n, 1 / n) \mid n=2,3,4, \cdots\}$ is closed in $R \times R$ but its image in $Y \times Y$ is not closed.) In fact, by Theorem $\mathrm{B}$ below, $Y \times Y$ is not the closed, continuous image of any metric space.

THEOREM B. If $X$ and $Y$ are nondiscrete topological spaces and if $X \times Y$ is the closed, continuous image of a metric space, then $X \times Y$ is metrizable.

We shall prove Theorem B, using Theorem A.

2. Semicanonical covers. Let $\vartheta$ be a collection of subsets of a set $S$. For each $W \subset S$, we define the star of $W$ with respect to $v$ by

$$
\operatorname{st}(W, v)=U\{V \in v \mid W \cap V \neq \varnothing\} .
$$

A pair $(Y, B)$ is a topological space $Y$ together with a closed subset $B$. If $Y$ is metrizable, then $(Y, B)$ is called a metric pair.

Let $(Y, B)$ be a pair. As in [1], we call a collection $V=\left\{V_{\alpha}\right\}$ of open subsets of $Y$ a semicanonical cover for $(Y, B)$ if

(1) $U_{\alpha} V_{\alpha}=Y-B$, and

(2) for each $b \in B$ and each neighborhood ${ }^{1} U$ of $b$ in $Y$ there exists

Received by the editors April 1, 1968.

${ }^{1}$ All neighborhoods are open. 
a neighborhood $W$ of $b$ in $Y$ such that $\operatorname{st}(W, v) \subset U$.

If a semicanonical cover exists for $(Y, B)$, we call $(Y, B)$ a semicanonical pair.

Lemma 1. Every metric pair $(Y, B)$ is semicanonical.

Proof. Let $d$ be a metric for $Y$. For each $y \in Y-B$, let $V_{y}$ $=\left\{x \in Y \mid d(x, y)<\frac{1}{2} d(y, B)\right\}$. Then the collection $\left\{V_{y} \mid y \in Y-B\right\}$ is a semicanonical cover for $(Y, B)$.

Lemma 2. Let $v$ be a semicanonical cover for a pair $(Y, B)$, and let $C \subset B$. If $U$ is a neighborhood of $C$ in $Y$, then there exists a neighborhood $W$ of $C$ in $Y$ such that $\operatorname{st}(W, v) \subset U$.

Proof. Each $x \in C$ has a neighborhood $W_{x}$ in $Y$ such that $\operatorname{st}\left(W_{x}, v\right)$ $C U$. Take $W=U_{x \in C} W_{x}$.

Suppose $f: X \rightarrow Y$ is a continuous closed surjection. A set $T \subset X$ is said to be saturated if $f^{-1} f(T)=T$. If $U \subset X$ is a neighborhood of a saturated set $T$, then there exists a saturated neighborhood $V$ of $T$ such that $V \subset U$. $\left\{\right.$ Proof: Take $V=f^{-1}(Y-f(X-U))$.

Lemma 3. Let $f: X \rightarrow Y$ be a continuous closed surjection, and suppose that $B$ is a closed subset of $Y$. If $\left(X, f^{-1}(B)\right)$ is a semicanonical pair, then $(Y, B)$ is a semicanonical pair.

Proof. Let $A=f^{-1}(B)$, and let $v$ be a semicanonical cover for $(X, A)$. For each $y \in Y-B$, let $G_{y}$ be a saturated neighborhood of $f^{-1}(y)$ in $X$ such that

$$
G_{y} \subset \operatorname{st}\left(f^{-1}(y), v\right)
$$

We shall show that

$$
\mathcal{G}=\left\{f\left(G_{y}\right) \mid y \in Y-B\right\}
$$

is a semicanonical cover for $Y-B$. Since $f$ is a closed surjection and since $G_{y}$ is open and saturated, $f\left(G_{y}\right)$ is open in $Y$, and it is obvious that

$$
Y-B=\bigcup\left\{f\left(G_{y}\right) \mid y \in Y-B\right\} .
$$

It remains to show that if $b \in B$ and if $U$ is a neighborhood of $b$ in $Y$, then there exists a neighborhood $U_{0}$ of $b$ such that $\operatorname{st}\left(U_{0}, \mathcal{G}\right) \subset U$. By Lemma $2, f^{-1}(b)$ has a neighborhood $W$ in $X$ such that $\operatorname{st}(W, v)$ $C f^{-1}(U)$. Since $f$ is a continuous closed surjection, we may choose $W$ to be saturated. Similarly, there exists a saturated neighborhood $W_{0}$ of $f^{-1}(b)$ in $X$ such that $\operatorname{st}\left(W_{0}, v\right) \subset W$. The set $U_{0}=f\left(W_{0}\right)$ is a neighborhood of $b$ in $Y$. We claim that $\operatorname{st}\left(U_{0}, \mathcal{G}\right) \subset U$. For suppose that 


$$
f\left(G_{y}\right) \cap U_{0} \neq \varnothing \text { for some } y \in Y-B .
$$

Then, since $G_{\nu}$ and $W_{0}$ are saturated and $f$ is surjective,

$$
G_{y} \cap W_{0} \neq \varnothing .
$$

By (1) and (2), there exist a point $x \in f^{-1}(y)$ and a $V \in \mathcal{V}$ such that $x \in V$ and $V \cap W_{0} \neq \varnothing$. Since $\operatorname{st}\left(W_{0}, v\right) \subset W$,

$$
V \subset W \text {. }
$$

Because $W$ is saturated and $x \in f^{-1}(y) \cap V$, it follows from (3) that $f^{-1}(y) \subset W$. Since st $(W, v) \subset f^{-1}(U)$, we have in particular

$$
\operatorname{st}\left(f^{-1}(y), v\right) \subset f^{-1}(U) .
$$

It follows from (4) and (1) that $f\left(G_{y}\right) \subset U$. Therefore, $\operatorname{st}\left(U_{0}, \mathcal{S}\right) \subset U$, and the proof is complete.

3. Proof of Theorem B. Suppose $f: M \rightarrow X \times Y$ is a continuous closed surjection, where $M$ is metrizable. Since $Y$ is homeomorphic to a closed subset of $X \times Y$, it follows that $Y$ is the image of a metric space under a continuous closed surjection. Consequently, if $y^{*}$ is an accumulation point of $Y$, then there exists a sequence $y_{1}, y_{2}, \ldots$ in $Y-\left\{y^{*}\right\}$ such that

$$
\lim _{n \rightarrow \infty} y_{n}=y^{*}
$$

We shall show that $X$ is first-countable. Let $x \in X$. By Lemmas 1 and $3,\left(X \times Y, X \times\left\{y^{*}\right\}\right)$ is a semicanonical pair; let $v$ be a semicanonical cover for it. For each positive integer $n$, choose $V_{n} \in V$ such that $\left(x, y_{n}\right) \in V_{n}$. Define $U_{n}=\pi\left(V_{n}\right)$, where $\pi: X \times Y \rightarrow X$ is the coordinate projection. We claim that $\left\{U_{n} \mid n \geqq 1\right\}$ is a basis for the neighborhoods of $x$ in $X$. To show this, let $U$ be a neighborhood of $x$ in $X$, and let $W$ be a neighborhood of $\left(x, y^{*}\right)$ in $X \times Y$ such that $\operatorname{st}(W, v)$ $C \pi^{-1}(U)$. It follows from (1) that there exists an integer $n$ such that $W \cap V_{n} \neq \varnothing$. Therefore $V_{n} \subset \pi^{-1}(U)$, and this implies that $U_{n} \subset U$. It follows that $X$ is first-countable.

Similarly, $Y$ is first-countable. Therefore $X \times Y$ is first-countable, and, by Theorem A, metrizable.

CoROLlaRY. Let $f: M \rightarrow X \times Y$ be a continuous closed surjection, where $M$ and $Y$ are metrizable and $Y$ is not discrete. Then $X \times Y$ is metrizable.

Proof. If $X$ is discrete, then it is metrizable, and therefore $X \times Y$ is metrizable. If $X$ is not discrete, the result follows from Theorem B. 


\section{REFERENCES}

1. D. M. Hyman, A generalization of the Borsuk-Whitehead-Hanner theorem, Pacific J. Math. 23 (1967), 263-271.

2. K. Morita and S. Hanai, Closed mappings and metric spaces, Proc. Japan Acad. 32 (1956), 10-14.

3. A. H. Stone, Metrizability of decomposition spaces, Proc. Amer. Math. Soc. 7 (1956), 690-700.

University of Southern California 